International Journal of Wireless \& Mobile Networks (IJWMN) Vol.2, No.4, November 2010

\title{
VOIP PERFORMANCE OVER BROADBAND WIRELESS NETWORKS UNDER STATIC AND MOBILE ENVIRONMENTS
}

\author{
Anindita Kundu ${ }^{1}$, Iti Saha Misra ${ }^{2}$, Salil K. Sanyal ${ }^{3}$, Suman Bhunia ${ }^{4}$, \\ Department of Electronics and Telecommunication Engineering, Jadavpur University, \\ Kolkata-700032, India

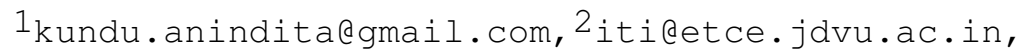 \\ 3 s_sanyaldieee.org, ${ }^{4}$ sumanbhunia@gmail.com
}

\begin{abstract}
Voice over IP is expected to be very promising application in the next generation communication networks. The objective of this paper is to analyse the VoIP performance among the most competing next generation wireless networks like WiMAX, WLAN and its integrated frameworks etc. WiMAX having higher bandwidth provides higher capacity but with degraded quality of service while WLAN provides low capacity and coverage. Hence, an integrated network using WiMAX backbone and WLAN hotspots has been developed and VoIP application has been setup. As OPNET 14.5.A provides a real life simulation environment, we have opted OPNET as the simulation platform for all performance studies in this work. Quality of the service is critically analysed with parameters like jitter, MOS and delay for various voice codecs in the aforesaid networks for both fixed and mobile scenario. Finally, it is observed and concluded that the WiMAX-WLAN integrated network provides improved and optimal performance over WLAN and WiMAX network with respect to network capacity and quality of service. Exhaustive simulation results have been provided.
\end{abstract}

\section{KEYWORDS}

WiMAX-WLAN Integrated network, backbone, hotspot, VoIP Codecs, Silence Suppression

\section{INTRODUCTION}

Wireless networking can now be considered as the backbone of the modern telecommunication system. The fast demand for high speed data transfer without appreciable loss has led to the evolution of technologies like WiMAX and WLAN. Although the above technologies have reached an enhanced level to meet the customer demand, still there is ample scope to increase the data rate and quality of service (QoS) beyond the present level.

WLANs [1] are mostly designed for private wired LANs and have been enormously successful for data traffic but not for voice traffic as it is highly sensitive to delay and loss [2]. Voice over WLAN has become popular, but maintenance of the speech quality is still one of many technical challenges of the VoIP system. VoIP is spreading rapidly and huge requirement of supporting multiple concurrent VoIP communications is coming up, but WLAN can support only a handful number of users [3] [4].

The IEEE 802.11 Standard supporting WLAN architecture specifies two different mechanisms, namely the contention-based Distributed Coordination Function (DCF) [5] and the pollingbased Point Coordination Function (PCF) [1] under MAC sub layer of the data link layer. DCF uses a carrier sense multiple access with collision avoidance (CSMA/CA) scheme for medium access and the optional four way handshaking request-to-send/clear-to-send [1] (RTS/CTS) DOI: $10.5121 /$ ijwmn.2010.2407 
mechanisms to eliminate hidden and exposed terminal problem. Best effort service can only provide satisfactory results based on traffic types in case of unavailability of differentiation and prioritization. However, for real time traffic such as VoIP the scheme does not support better QoS. These features make the DCF scheme a less feasible option to support QoS for VoIP traffic. On the other hand, the PCF mode enables the polled stations to transmit data without contending for the channel. Studies on VoIP over WLAN in PCF mode [6] show that the polling overhead is high with increased number of stations in a basic service set (BSS). This results in excessive delay and poor performance of VoIP under heavy load conditions in PCF mode. Thus, both DCF and PCF modes have limited support for real-time applications. Supporting VoIP over WLAN using DCF mode poses significant challenges, because the performance characteristics of their physical and MAC layers are much worse than their wired counterparts. Thus, there is sufficient reason to make a performance study for VoIP over WLAN.

WiMAX (Worldwide Interoperability for Microwave Access) [7][8] on the other hand is designed to deliver a metro area last mile Broadband Wireless Access (BWA) service. So, while wireless LAN supports transmission range of up to few hundred meters, WiMAX system ranges up to 30 miles [7]. Unlike a typical IEEE 802.11 WLAN with 11Mbps bandwidth which supports very limited VoIP connections [4], an IEEE 802.16 WiMAX with 70Mbps bandwidth [9] has the capacity to support comparatively large number of VoIP users. These features led to the study and comparison of the VoIP QoS in IEEE 802.11b WLAN and IEEE 802.16 WiMAX networks.

IEEE 802.16 support 5 types of service classes, namely UGS (Unsolicited Grant Service), rtPS (real time Polling Service), nrtPS (non-real time Polling Service), BE (Best Effort Service), ertPS (extended rtPS service) [10]. UGS supports fixed-size data packets at a constant bit rate (CBR). It supports real time applications like VoIP or streaming applications but wastes bandwidth during the off periods. rtPS supports variable bit rate(VBR) real-time service such as VoIP. Delay-tolerant data streams such as an FTP is designed to be supported by the nrtPS. This requires variable-size data grants at a minimum guaranteed rate. The nrtPS is similar to the rtPS but allows contention based polling. Data streams, such as Web browsing, that do not require a minimum service-level guarantee is supported by BE service. BE connections are never polled and receive resources through contention. ertPS was introduced to support VBR real-time services such as VoIP and video streaming. It has an advantage over UGS and rtPS for VoIP applications as it carries lower overhead than UGS and rtPS [11] and hence is modeled in our system.

Since, WiMAX is expected to create the opportunity to successfully penetrate the commercial barrier by providing higher bandwidth, establishing wireless commons becomes an important factor. The audible spectrum being fixed and a large part of the audible bandwidth already been allocated to some organisations, the requirement of bandwidth for other organisations is still increasing [12]. This has resulted in the bandwidth crunch. Also, with the fourth generation mobile networks taken into consideration, network integration is another major technical and social challenge regarding the future of the community-based Wi-Fi networks [13]. According to [13], the foundation of the WiMAX PTP commons is the process of hot-spot interconnection and integration. Instead of global Internet connectivity, many current applications and businesses are expected to be better utilized with decreased bandwidth consumption by the use of localized Wi-Fi constellation. With a step towards the next generation, an integrated network as shown in Figure 1, comprising of both the WiMAX and WLAN network and using nodes with dual stack is expected to provide a better performance with respect to the QoS and bandwidth utilisation than a single WiMAX or WLAN network.

VoIP has been widely accepted for its cost effectiveness and easy implementation. A VoIP system consists of three indispensable components, namely 1) codec, 2) packetizer, and 3) playout buffer. Analog voice signals are compressed, and encoded into digital voice streams by 
the codecs. The output digital voice streams are then packed into constant-bit-rate (CBR) voice packets by the packetizer. Voice signal when transmitted over an IP network, is first divided into small fixed or variable size pieces, called payloads. One or more of these pieces are then packed together for transmission. These packs are further encapsulated using one or more appropriate sets of headers to generate IP packets for transmission. The packet header contains all information pertaining to the destination, routing, control, and management of the IP packets [14].Thus, preset circuits for transmission of information are not required. However, additional bandwidth, processing, and memory space is needed for the packet header processing, and packet buffering. This is an essential factor to be considered for real time applications like VoIP. The smaller the number of voice or speech frames packed into a packet, the greater the protocol/encapsulation overhead and processing delay. The larger the number of voice or speech frames packed into one packet, the greater the packet processing or storing and transmission delay [14]. The playout buffer is used at the receiver end to smooth the speech by removing the delay jitter. Additional network delay not only causes the receiver's playout buffer to wait longer before reconstructing voice signal, it can also affect the liveliness of a speech signal during a telephone conversation. ITU-T's G.114 [15] states that the one-way ETE delay must be less than $150 \mathrm{msec}$, and the packet loss must remain low (e.g., less than 5\%) in order to maintain the toll quality of the voice signal [16]. A one way conversation is very sensitive to packet delay jitter but can tolerate certain (about 5\%) of packet loss [15]. Loss of large number of contiguous packets may give the impression of connection dropout to the communicating parties.

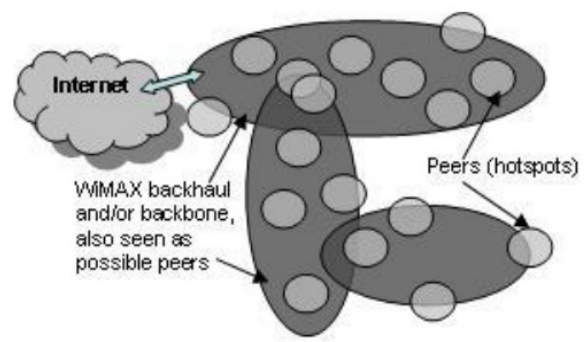

Figure 1. WLAN integration using WiMAX [13]

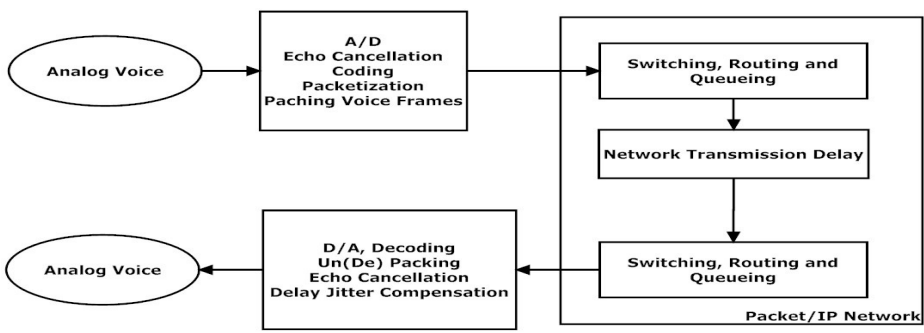

Figure 2. A high-level packet voice transmission model [14]

The schematic diagram of a simple high-level packet voice transmission model is shown in Figure 2. At the outgoing side, the analog voice signal is first digitized and packetised (voice frame) using the techniques presented before. One or more voice frames are then packed into one data packet for transmission. This involves mostly UDP encapsulation of RTP packets. The UDP packets are then transmitted over a packet-switched (IP) network. This network adds (a) switching, routing, and queuing delay, (b) delay jitter, and (c) probably packet loss. At the incoming side, in addition to decoding, deframing, and depacking, a number of data/packet processing mechanisms need to be incorporated to mitigate the effects of network impairments such as delay, loss, delay jitter, etc. The objective is to maintain the real-timeliness, liveliness, or interactive behavior of the voice streams. 
International Journal of Wireless \& Mobile Networks (IJWMN) Vol.2, No.4, November 2010

Quality of VoIP transmission under noisy environment is usually measured in terms of jitter [17], MOS [18] and packet end-to-end delay [19]. Perceived voice with zero jitter, high MOS and low packet end-to-end delay is considered to be the best. With the two competing wireless networks namely WLAN and WiMAX, this paper analyses the perceived voice quality as measured using OPNET 14.5.A and 15.0.A simulation environment.

\section{VOICE OVER IP}

Voice is analog and is converted into the digital format before transmitting over Internet. This process is called encoding and the reverse is called decoding. Both the tasks are performed by voice codecs [20]. With bandwidth utilization becoming a great concern, voice compression techniques are used in practice [20] to reduce the bandwidth consumption. Voice compression by a codec adds an additional overhead of algorithmic delay. Thus, a codec is expected to provide good quality of VoIP even after compression, with minimum delay.

Table 1 shows the bandwidth requirements of some common codecs. G.711 is the international standard for encoding telephone audio. It has a fixed bit rate of 64kbps. G.729 and G.723.1 are low bit rate codecs at the expense of high codec complexity. G.729 is an industry standard with high bandwidth utilization for toll-quality voice calls [21]. G.723.1 is one of the most efficient codecs with the highest compression ratio and is usually used in video conferencing applications. G.726 uses ADPCM speech codec standard, and transmits at rates of 16, 24, 32, and 40 kbps. G.728 officially codes speech at 16kbps using low-delay code excited linear prediction [22]. For example, during a call using G.711 as codec, the amount of data transfer for both uplink and downlink will be $87.2 \times 2=174.4 \mathrm{kbps}=0.1703 \mathrm{Mbps}=10.21 \mathrm{Mb}$ per minute. So, G.711 uses $10.21 \mathrm{Mb} / \mathrm{min}$ per VoIP call where as G.729 uses $0.5 \mathrm{Mb} / \mathrm{min}$ per voice call in the same way.

Table 1. Bandwidth Requirement of Some Common Codecs [20] [23]

\begin{tabular}{llll}
\hline Codecs & Algorithm & $\begin{array}{l}\text { Bandwidth } \\
(\mathrm{kbps})\end{array}$ & $\begin{array}{l}\text { Ethernet } \\
\text { Bandwidth } \\
\text { Usage } \\
(\mathrm{kbps})\end{array}$ \\
\hline G 711 & PCM(Pulse Code Modulation) & 64 & 87.2 \\
G 729 & $\begin{array}{l}\text { CS-ACELP (Conjugate Structure Algebraic-Code } \\
\text { Excited Linear Prediction) }\end{array}$ & 8 & 31.2 \\
G 723.1 & Multi Rate Coder & 6.3 & 21.9 \\
G 723.1 & Multi Rate Coder & 5.3 & 20.8 \\
G 726 & ADPCM(Adaptive Differential Pulse Code Modulation) & 32 & 55.2 \\
G 726 & ADPCM(Adaptive Differential Pulse Code Modulation) & 24 & 47.2 \\
G 728 & LD-CELP (Low-Delay Code Excited Linear Prediction) & 16 & 31.5 \\
\hline
\end{tabular}

Moreover, recently voice codecs are so developed to detect additional parameters talk-spurt [24] and silence lengths [24] within a conversation. During a voice communication the time duration during which the user speaks is called a talk-spurt and the time duration during which the user is silent is called the Silence length or gap. Silence within a communication period leads to the packetization of the background noise and sending it over the network. This causes bandwidth wastage. Usually, during a conversation we talk $35 \%$ of the time and remain quiet rest of the 
time [24]. Silence suppression is done by the voice activity detection (VAD) algorithm. With silence suppression during the silence period, the codec does not send any voice data. Instead of voice data the VAD algorithm generates comfort noise which has packet size much less than the voice traffic [14]. This is shown in figure 3a. This decreases channel utilisation and thereby saves bandwidth. Figure $3 \mathrm{~b}$ shows the scenario when silence is not suppressed and the background noise are packetised along with the voice signal.

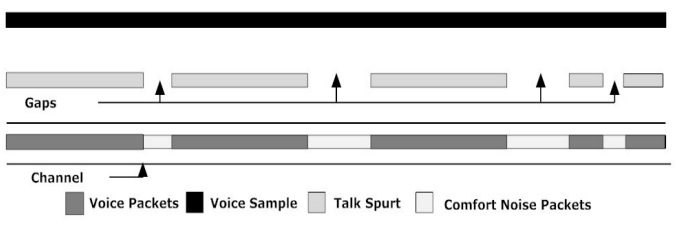

(a)

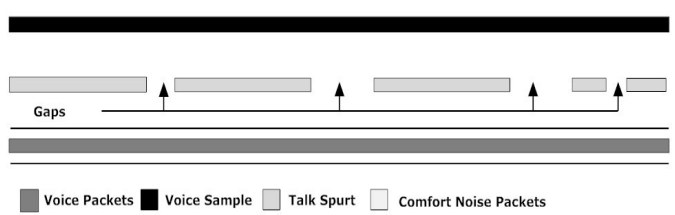

(b)

Figure 3. Packet Creation based on voice activity. (a) Silence is suppressed and Comfort Noise Generated (b) Silence is not suppressed.

Communication over wireless medium is noise sensitive. Noise causes the signal to reach the destination with a lead or lag in time. This deviation is called jitter. Lead causes negative jitter and lag causes positive jitter and both degrade the voice quality. The time taken by voice to be transmitted from the mouth of the sender to the ear of the receiver is called packet end-to-end delay. The packet end-to-end delay should be within $150 \mathrm{~ms}$ for one way voice communication [14]. Perceived voice quality is typically estimated by the subjective MOS, an arithmetic average of opinion score. MOS of a particular codec is the average mark given by a panel of auditors listening to several recorded samples. It ranges from 1(unacceptable) to 5 (excellent). It depends on delay and packet dropped in the network. The E-model, an analytical model defined in ITU-T recommendation[25], provides a framework for an objective on-line quality estimation based on network performance measurements like delay and loss and application level factors like low bit rate codecs. The result of the E-model is the calculation of the R-factor (best case 100 worst case 0) [25] is given by,

$$
R=R_{0}-I_{s}-I_{d}-I_{e}+A .
$$

where the term $\mathrm{R}_{0}$ expresses the basic signal-to-noise ratio (received speech level relative to circuit and acoustic noise), $\mathrm{I}_{\mathrm{s}}$ represents all impairments that occur more or less simultaneously with the voice signal, such as: too loud speech level, non-optimum side tone, quantization noise, etc., $I_{d}$ represents the impairment caused due to delay and echo effects, $I_{e}$ is an "effective equipment impairment factor", which represents impairments caused by low bit-rate codecs. It also includes impairment due to packet-losses of random distribution. The advantage factor ' $A$ ' allows for an "advantage of access" for certain systems relative to conventional systems, trading voice quality for convenience. While all other impairment factors are subtracted from the basic signal-to-noise ratio $R_{0}$, ' $A$ ' is added and thus compensates other impairments to a certain amount. It can be used to take into account the fact that the user will tolerate some decrease in transmission quality in exchange for the "advantage of access". Examples of such advantages are cordless and mobile systems or connections into hard-to-reach regions via multi satellite hops. ' $A$ ' is 10 for mobile telephony but 0 for VoIP [6]. $R_{0}$ is considered to be 94.77 and $I_{s}$ is considered to be 1.43 in OPNET 14.5.A. The relation between MOS and R-factor:

$$
\text { MOS }=1+0.035 \mathrm{R}+7.10-6 \mathrm{R}(\mathrm{R}-60)(100-\mathrm{R})
$$

The purpose of this modeling is to compare the performance parameters for the voice codecs in WiMAX 802.16, WLAN 802.11b and their integrated network and thereby to show that the integration provides optimal network capacity and quality of service. 
International Journal of Wireless \& Mobile Networks (IJWMN) Vol.2, No.4, November 2010

\section{Simulation EnVironment}

\subsection{Scenario 1: Fixed network}

Figure 4a shows the fixed WiMAX network setup. The Wireless Deployment Wizard of OPNET is used to deploy a 7 celled WiMAX network, with multiple subscriber stations under the coverage of a base station.

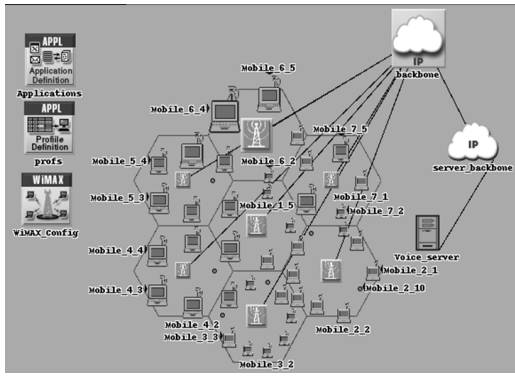

(a)

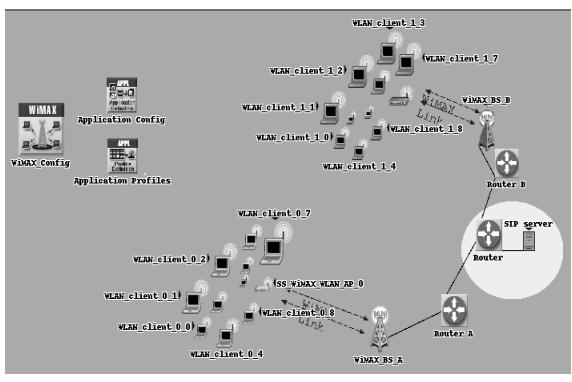

(b)

Figure 4. Model for (a) Fixed WiMAX (b) WiMAX-WLAN Integrated Network

The base station is connected to the core network by a server backbone via an IP backbone and an ASN Gateway which controls the mobility of the mobile nodes. The server backbone is further connected to the voice server, configured as the SIP server. The base station, IP Backbone, Server Backbone, ASN Gateway and the Voice Server together represent the service provider company network. The Base Station and subscriber station parameters are as shown in Table 2. The number of subscribers in cell 2 and cell 3 are 10 and VoIP calls are configured between them in mesh.

Table 2. WiMAX and WLAN Network Parameters

\begin{tabular}{|c|c|c|c|c|}
\hline $\begin{array}{l}\text { WiMAX } \\
\text { Parameters }\end{array}$ & Values & & $\begin{array}{l}\text { WLAN } \\
\text { Parameters }\end{array}$ & Values \\
\hline $\begin{array}{ll}\text { WiMAX } & \text { Service } \\
\text { Class } & \end{array}$ & ertPS & & $\begin{array}{l}\text { Physical } \\
\text { Characteristics }\end{array}$ & $\begin{array}{ll}\text { Direct } & \text { Sequence } \\
\text { (DSSS) } & \end{array}$ \\
\hline $\begin{array}{l}\text { BS Transmission } \\
\text { Power }\end{array}$ & $10 \mathrm{~W}$ & & Data Rate & $11 \mathrm{Mbps}$ \\
\hline $\begin{array}{l}\text { SS Transmission } \\
\text { Power }\end{array}$ & $0.5 \mathrm{~W}$ & & $\begin{array}{l}\text { Transmission } \\
\text { Power }\end{array}$ & $5 \mathrm{~mW}$ \\
\hline PHY profile & $\begin{array}{l}\text { Wireless } \\
\text { 20MHz }\end{array}$ & OFDMA & Buffer Size & 2048000 bits \\
\hline
\end{tabular}

Similar to the WiMAX network, a WLAN 802.11b network is also deployed by the Wireless Deployment Wizard of OPNET featuring a 7 celled WLAN network with multiple subscriber stations. Unlike, the WiMAX network, the WLAN network has Access Points (APs) in place of the base stations and the mobile nodes of WiMAX are replaced by mobile nodes of WLAN. The APs are also connected to the core network. The APs are connected to the voice server configured as the SIP server via an IP backbone and a server backbone. These nodes represent the service provider company network in WLAN environment. Similar to the WiMAX network, the VoIP calls are setup between the subscribers of cell 2 and cell 3 in mesh. The important parameters of the access points and the subscriber stations are as shown in Table 2.

Like Figure 1, a WLAN integrated network using WiMAX is developed as shown in Figure 4b. Two WiMAX Base Stations are connected to each other and a SIP server via routers. A special 
type of node called SS_WiMAX_WLAN_AP having dual stack of both WiMAX and WLAN as shown in Figure 5, is configured as the subscriber station of WiMAX network and Access Point for the WLAN network. This node is the bridge between the WLAN subscriber stations and the WiMAX base station. There are 10 WLAN subscriber stations under each SS_WiMAX_WLAN_AP. VoIP calls are setup between 10 users of two such SS_WiMAX_WLAN_AP. The parameters of the WiMAX Base Station and the WLAN subscriber stations are same as shown in Table 2.

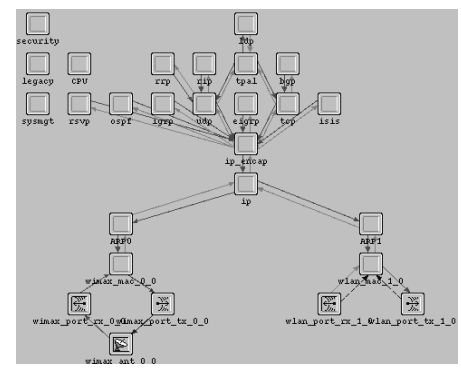

Figure 5. Protocol Stack of SS_WiMAX_WLAN_AP

\subsection{Scenario 2: Mobile Network}

Figure 6a shows the simulation setup used for mobile WiMAX network. Here, Generic Routing Encapsulation (GRE) tunnels are setup between the ASN gateway and the base stations to control the mobility of the mobile nodes. The mobile nodes are configured to move at a rate of $50 \mathrm{~km} / \mathrm{hr}, 100 \mathrm{~km} / \mathrm{hr}$ and $150 \mathrm{~km} / \mathrm{hr}$. The number of subscribers in cell 2 is 10 and the same in cell 3 and 7 are 5 each. Voice over IP calls are setup between them using SIP and are shown by the blue lines in the Figure 6.

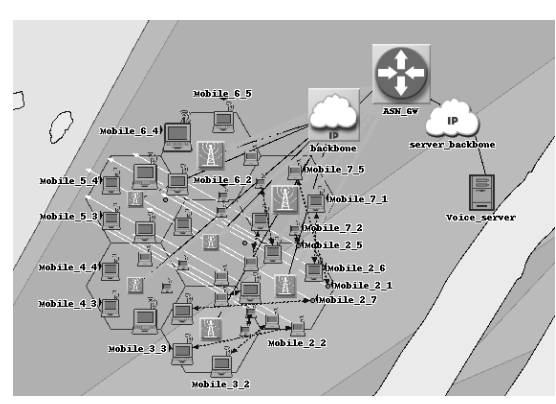

(a)

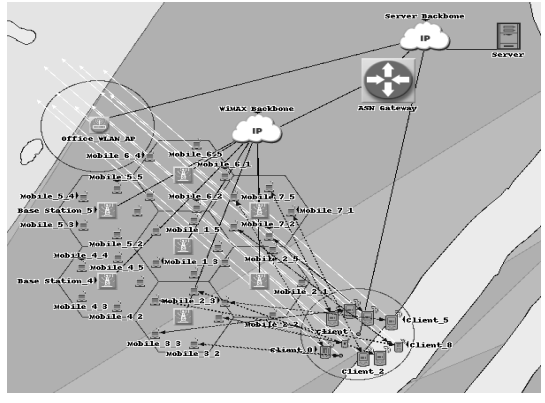

(b)

Figure 6. Model for (a) Mobile WiMAX (b) Mobile WiMAX-WLAN Integrated Network

Similarly, a WLAN 802.11b network is also deployed and mobility is configured. Another scenario having two WLAN hot spots and a large span of area between the hot spots covered by WiMAX is setup. Such a scenario is shown in Figure 6b. The WiMAX part of the network is deployed by the Wireless deployment wizard of OPNET and WLAN APs are obtained from the object palette. 10 special nodes are configured to move from one AP to the other i.e. from one hot spot to another hot spot while communicating with 5 nodes of cell number 3 and 5 nodes of cell number 7 of the WiMAX network. Results show that the mobile nodes are supported and traffic is sent to them even when they are out of the coverage of the WLAN network and within the jurisdiction of WiMAX network. 
International Journal of Wireless \& Mobile Networks (IJWMN) Vol.2, No.4, November 2010

\section{Simulation Results AND Discussion}

\subsection{Scenario: Fixed Network}

The variation of jitter with and without silence suppression is shown in Figure 7. From 7a, we see that the average voice jitter is almost 0 for WiMAX implying very good quality of voice while WLAN has a positive jitter varying from about 0.0007 to 0.001 seconds. The integrated network shows jitter variation from about 0.0004 to 0.0006 seconds. For G.723.1, the average jitter is almost 0 irrespective of the network indicating a very good performance. This is because the bit rate of G.723.1 is 6.3 or $5.3 \mathrm{kbps}$ which results in generation of small packets. However, modem and fax signals cannot be carried by G.723.1 [24]. It can be used only for narrow band communications.

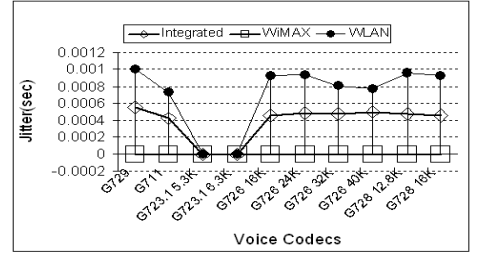

(a)

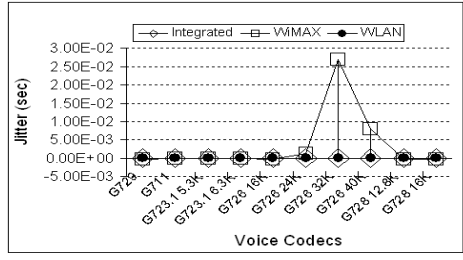

(b)

Figure. 7. Average jitter, (a) Without silence suppression (b) With silence suppression

With silence suppression, as shown in Figure 7b, the result is different. Like G.711, G.726 has its roots in the PSTN network. It is primarily used for international trunks to save bandwidth. Unlike G.711, G.726 uses 32 kbps to provide nearly the same quality of voice [26] because 32 kbps is the de facto standard. As shown in Figure 7b, the average voice jitter is almost 0 for all codecs in both WLAN and WLAN-WiMAX integrated network where as the WiMAX network shows a slight deviation for the voice codec G.726. The deviation for G.726 with 32kbps is the highest and is about $0.03 \mathrm{sec}$ which is within the allowable limit. G.726 also supports data rates of $16 \mathrm{kbps}$, 24kbps, and 40kbps. The $24 \mathrm{kbps}$ and $16 \mathrm{kbps}$ channels are used for voice communication in Digital Circuit Multiplication Equipment (DCME) and the $40 \mathrm{kbps}$ is used for data modem signals (especially modems doing $4800 \mathrm{kbps}$ or higher) in DCME.

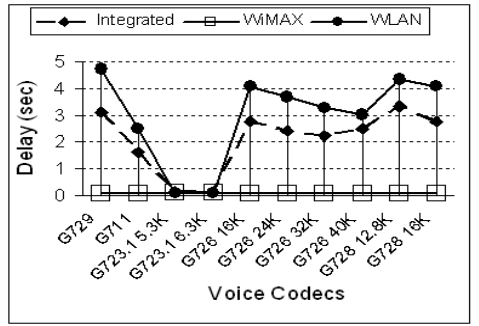

(a)

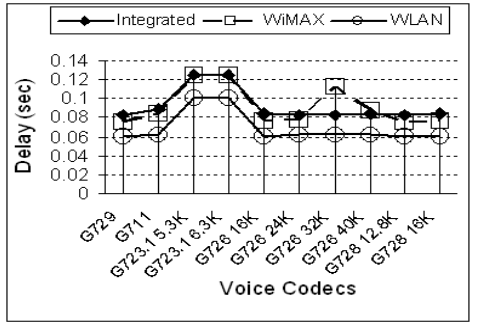

(b)

Figure. 8. Average Packet End-to-end Delay (a) Without silence suppression (b) With silence suppression

Figure 8 shows the packet end-to-end delay variation of the delay sensitive VoIP application for the three networks. As shown in Figure 8a, the packet end-to-end delay for voice without silence suppression is less than 0.5 seconds for WiMAX network. On the other hand, the high bit rate codecs of WLAN network has very high packet end-to-end delay. This is because the silence period is also packetized which results in an increase in the demand for bandwidth requirement and causes congestion in the WLAN network. Since, the integrated network has a 
higher capacity; it shows reduced delay than the WLAN network as increased capacity results in lower congestion.

As shown in Figure 8b, the packet end-to-end delay for VoIP with silence suppression for WiMAX network is more than WLAN network while for the integrated network it is almost the same as that in the WiMAX network. With silence suppression, the number of packets to be sent decreases and thereby releases the congestion in the WLAN network. The distance to be traversed by the packet in the WiMAX network is much higher than that to be covered in the WLAN network. Even for the integrated network, the distance traversed by the packet is almost as large as that of WiMAX network. For WLAN, the packet end-to-end delay is about 0.06 seconds except for G.723.1 where as for WiMAX and WLAN-WiMAX integrated network, the delay varies from 0.08 seconds (for G.729 and G.711) to 0.13 seconds (for G.723.1).

Figure 9 shows the average MOS obtained by the three networks. As mentioned before, MOS depends on the packet end-to-end delay and packets loss. As the Figure 9a shows, MOS value obtained for WiMAX it is above 3, for WLAN it is almost 1. For the integrated network the MOS value is about 1.5 except for G.723.1 for which it is 2.5. G.723.1 being a low bit rate codec creates numerous packets which results in increased delay due to packet reassembly. As a result the MOS value is low in case of WiMAX network. On the other hand, G.723.1 has low bandwidth requirement and hence less packets are dropped compared to the other codecs in WLAN network. Hence it has higher MOS compared to the other codecs.

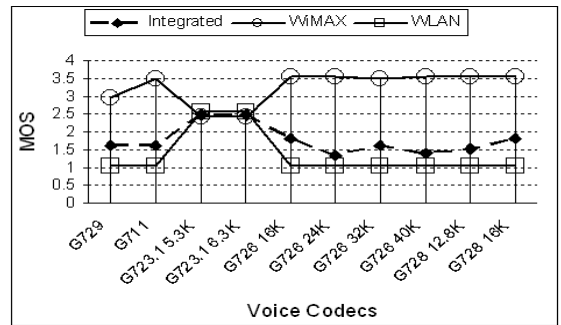

(a)

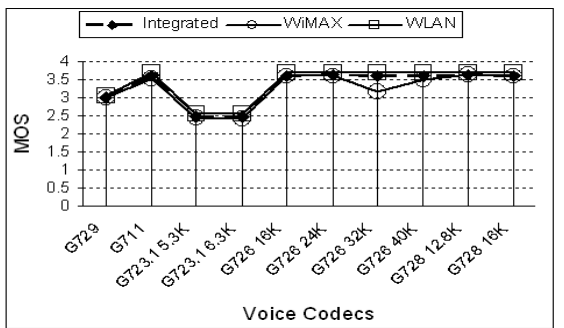

(b)

Figure. 9. Average voice MOS (a) Without silence suppression (b) With silence suppression

The result with silence suppression is totally different as is shown in Figure 9b. Here, WLAN network performs the best among the three networks with a MOS value above 3.5 except for G.711 and G.723.1. The MOS value for G.711 is about 3 and for G.723.1 is about 2.5. With the silence suppression, the number of packets in the network decreases which releases the congestion in the WLAN network. This reduces the number of packets dropped and hence increases the MOS value. The codec G.723.1 is a low bit rate codec having bit rate of $5.3 \mathrm{kbps}$ and $6.3 \mathrm{kbps}$. The packet end-to-end delay is larger for G.723.1 compared to the other codecs and this result in lower MOS values.

\subsection{Scenario 2: Mobile Network}

Figure 10 shows the variation of jitter for all the three networks in the mobile scenario. The mobile nodes are configured to move at a speed of $50 \mathrm{~km} / \mathrm{hr}, 100 \mathrm{~km} / \mathrm{hr}$ and $150 \mathrm{~km} / \mathrm{hr}$. As shown in the figure, the average jitter for WLAN network is very high compared to the WiMAX network. The WLAN-WiMAX integrated network shows variation of jitter almost like that of WiMAX. The jitter is very low compared to WLAN implying very good performance. The average performance of the networks with respect to the variations of speed of the mobile nodes is almost the same. Between the integrated network and the WiMAX network, the WiMAX network performs slightly better as no vertical handover is involved. Average Jitter with silence suppression could not be analyzed due to the limitations of OPNET. 


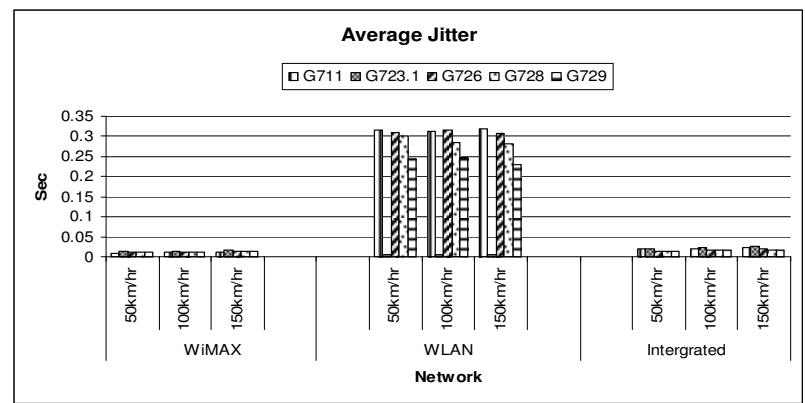

Figure. 10. Average Jitter in mobile network

As shown in Figure 11a, the average packet end-to-end delay for the WiMAX network and WLAN-WiMAX integrated network is negligible compared to the WLAN network. The delay shown by the WLAN is in the order of minutes which render the network practically unusable. Figure $11 \mathrm{~b}$ shows the variation of delay between the WiMAX network and the integrated network which is almost zero with respect to WLAN. Between the two networks, WLAN is once again found to perform the best and the integrated network performance is slightly degraded. It is also observed that the delay increases with the speed and G. 711 has highest delay among other codecs for integrated network. This is because G. 711 is the highest bit rate codec and has a data rate of $64 \mathrm{kbps}$. As the mobile nodes move, they get handed over both horizontally and vertically. With high data rate, more packets eventually get dropped during the handover. Any packet dropped by the lower layer is perceived as delay by the upper layers. Hence, the delay associated with G .711 as exhibited in Figure 11b.

Close observation of the results reveals that WiMAX network performs better on the basis of jitter, MOS and packet end-to-end delay than conventional WLAN 802.11b in case of voice applications when no silence suppression is considered, i.e. with no bar on the bandwidth usage. However, when more users are to be accommodated, bandwidth becomes a constraint for which silence suppression has to be used. Now, with silence suppression, WLAN is observed to provide a better voice quality than WiMAX with the limitation of not supporting vehicular mobility. WiMAX network though provides high capacity with degradation of the voice quality as reflected from the MOS value, has to be used to support vehicular mobility. On the other hand WLAN has less capacity but provides a better voice quality in static scenario. The integrated network in the static scenario performs better than WiMAX and almost like WLAN i.e. holds the second best position and in the mobile scenario; the integrated network performs almost like WiMAX which is much better than WLAN. This is exhibited in Table 3. The mobile scenario with silence suppression could not be simulated due to the limitations of OPNET. However, the literature states that the capacity of mobile WiMAX network is doubled when silence suppression is used [27]. Hence, the integrated deployment of WiMAX and WLAN is expected to be competent enough to provide optimal voice quality with optimal network capacity in both static and mobile scenario. 
International Journal of Wireless \& Mobile Networks (IJWMN) Vol.2, No.4, November 2010

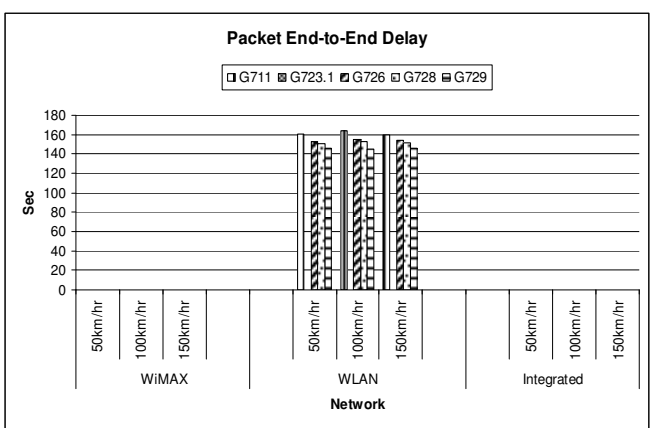

(a)

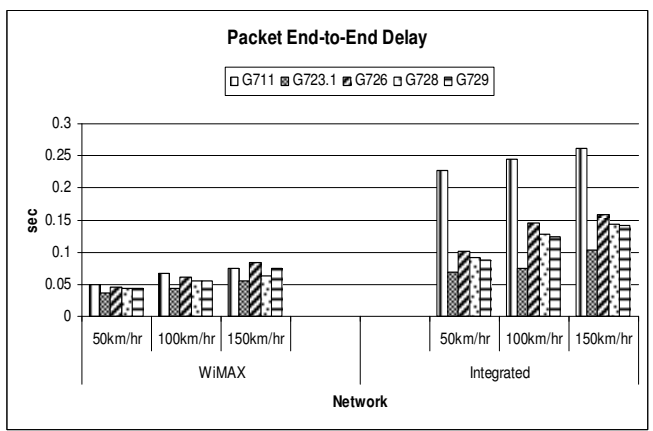

(b)

Figure. 11. Average Packet End-to-End Delay

Table 3. Tabular representation of the network performance and capacity

\begin{tabular}{|l|l|l|l|}
\hline & \multicolumn{2}{|l|}{ Static Network } & Mobile Network \\
\cline { 2 - 4 } & $\begin{array}{l}\text { With silence } \\
\text { suppression }\end{array}$ & $\begin{array}{l}\text { Without silence } \\
\text { suppression }\end{array}$ & $\begin{array}{l}\text { Without silence } \\
\text { suppression }\end{array}$ \\
\hline $\begin{array}{l}\text { WLAN-WiMAX } \\
\text { Integrated Network }\end{array}$ & Moderate & Moderate & Moderate \\
\hline WLAN & Good & Bad & Bad \\
\hline WiMAX & Bad & Good & Good \\
\hline
\end{tabular}

\section{Conclusion}

In this paper extensive simulation based performance analysis has been done over a WiMAX based BWA network, a WLAN (IEEE 802.11b), and also on a WLAN-WiMAX integrated network for VoIP application. For this purpose OPNET 14.5.A and 15.0.A have been used as the simulation platform. Multiple competing traffic sources using SIP signalling over the networks are generated and the trace of traffic and measurements for different performance parameters discussed in this paper are obtained for both fixed and mobile scenarios. Since, the work is focussed on VoIP application, the performance evaluation is carried out through different voice codecs as supported by OPNET and the measurement attribute is based on the performance parameters such as jitter, MOS, end-to-end delay etc. suitable for judging the quality of the network handling real time traffic. A comparative study for the three different network models for the above measurement has also been made for both scenarios. The optimal performance is exhibited by WiMAX-WLAN integrated network.

Acknowledgment: The authors deeply acknowledge the support from DST, Govt. of India for this work in the form of FIST 2007 Project on "Broadband Wireless Communications" in the Department of ETCE, Jadavpur University.

\section{References}

[1] B. P. Crow, I. Widjaja, J. G. Kim \& P.T.Satkai, (1997) "IEEE 802.11 Wireless Local Area Networks", IEEE Communications, IEEE Communications Magazine, vol. 35, no. 9, pp. 116126.

[2] D. Malone, P. Clifford, D.J. Leith, (2006) "On Buffer Sizing for Voice in 802.11 WLANs", IEEE Communications Letters, 10(10), 700-703.

[3] http://voip.biz-news.com/news/tags/en_US/VoIP 
International Journal of Wireless \& Mobile Networks (IJWMN) Vol.2, No.4, November 2010

[4] A Trapeze Networks White Paper,( 2006) "Smart MobileTM: Next-Generation Wlan Architecture For High Performance Networks".

[5] Y.Li, C.Wang, K. Long \& W. Zhao, "Modelling Channel Access Delay and Jitter of IEEE 802.11 DCF", Proceedings of Wireless Personal Communications, Vol 47, No. 3, pp. 417-440.

[6] D.Y. Chen, S. Garg, M. Kappes, \& K. S. Trivedi, (2002) "Supporting VBR VoIP traffic with IEEE 802.11 WLAN in PCF mode", Proceedings of OPNETWork 2002, Washington D. C.

[7] F.E.Retnasothie, M.K. Ozdemir , T.Y' Cek , H. Celebi , J. Zhang, \& R. Muththaiah, (2006)“Wireless IPTV over WiMAX: Challenges and Applications", Proceedings of IEEE WAMICON, pp 1-5,

[8] A. Ghosh, D. R. Wolter, J. G. Andrews \& R. Chen, (2005) "Broadband Wireless Access with WiMax/802.16: Current Performance Benchmarks and Future Potential", IEEE Communications Magazine, Vol 43, No. 2, pp. 129-136.

[9] L. X. Cai, X. (Sherman) Shen, J. W. Mark, L. Cai \& Y. Xiao, (2006) "Voice Capacity Analysis of WLAN With Unbalanced Traffic", IEEE Transations on Vehicular Technologies, Vol. 55, No.3.

[10]N.Nagarajan' , Rajeev \& B. Kaarthick, (2009)“Performnance analysis of Video Conferencing and Multimedia application Services over WiMAX”, IEEE International Advance Computing Conference (IACC 2009), Patiala, India.

[11] G. Chu, D.Wang \& S. Mei, (2002) "A QoS Architecture for the MAC protocol of IEEE 802.16 BWA System,” IEEE 2002 ICC, Circuits and Systems, vol. 1, pp. 435 -439.

[12] K.B.Letalef \& Wei Zhang, (2009) "Cooperative Communication for Cognitive Radio", Proceedings of IEEE, Vol 97, No. 5.

[13] B.Angelov \& B.Rao, (2009) "The Progression of WiMAX Toward a Peer-to- Peer Paradigm Shift", Annual Review of Communications, Vol 59.

[14]B. Khasnabish, "Implementing Voice over IP", Wiley-Inter Science, Lexington, Massachusetts, USA.

[15] G.114 Recommendation, (1996) “One Way Transmission Time”, ITU-T, Geneva.

[16] IEEE, (1998) IEEE Network Magazine, IEEE Press/Publishers, New York, Vol. 12, No.1, January/ February.

[17] J.Chin, A.Cantoni, (1998)“Phase Jitter $\equiv$ Timing Jitter”, IEEE Communications Letters, Vol. 2, No. 2.

[18] M.Molina-Garcia, J.I.Alonso, (2009) "Evolution of Mean Opinion Score due to Handover Events in IEEE 802.11 WLAN Networks", Proceedings of Radio and Wireless Symposium, San Diego, CA, pp. 207-210.

[19] M.Baldi, Y.Ofek, (1998) "End to End Delay of videoconferencing over Packet Switched Networks", INFOCOM'98, Proceedings of Seventeenth Annual Joint Conference of IEEE Computer and Communications Society, San Francisco, CA, USA, Vol. 3, pp. 1084-1092.

[20] http://whirlpool.net.au/wiki/?tag=VoIP_Codecs

[21] http://en.wikipedia.org/wiki/G.726

[22] Trad, F. Munir, H. Afifi, (2006) "Capacity Evaluation of VoIP in IEEE 802.11e WLAN Environment", IEEE CCNC 2006 Proceedings.

[23] http://wiki.snom.com/Bandwidth_requirements_and_QoS

[24] O.Hersent, J.Petit, D.Gurle, (2005) IP Telephony- Deploying Voice-Over-IP Protocols, John Wiley \& Sons, Ltd.

[25] "The E-Model, a Computational Model for use in Transmission Planning”, (2005) ITU-T Rec G.107.

[26] http://www.voip-info.org/wiki/view/ITU+G.726S.

[27] Chakchai So-In, Raj Jain, Abdel-Karim Tamimi, (2010) "Capacity Evaluation of IEEE 802.16e Mobile WiMAX", Journal of Computer Systems Networks, Networks and Communications, Volume 2010. 